that erythrose 4-phosphate is an end product.

The longest of the chapters deals with isoenzymes of lactate dehydrogenase. This is fairly comprehensive. There is, however, no account of the quaternary structure of the lactate dehydrogenase of dogfish muscle, as determined by $\mathrm{X}$-ray techniques. It is perhaps unfortunate that the author attributes to Langvad the discovery that the malignant type of shift in isoenzyme pattern occurs in the morphologically unchanged cells in the neighbourhood of malignant tumours. In actual fact this was described considerably earlier by British authors. In this chapter it is pointed out that the absence of the slowest moving isoenzyme of lactate dehydrogenase from red blood cells is apparently not consistent with random reassociation of enzyme subunits. This gives the totally erroneous impression that experiments done in vitro apply to the situation in vivo, although evidence has been available for some time that this is certainly not the case. The clinical sections of this chapter are undoubtedly too brief and would not be of great help to a clinician interested in differential diagnosis.

In the chapter dealing with phosphatase isoenzymes, there are a number of important omissions. There is insufficient discussion of the relationship of intestinal alkaline phosphatase to $\mathrm{ABO}$ blood groups and nothing about the effect of fatty meals. There is only the very briefest discussion of the Regan and other isoenzymes, which occur in the serum of cancer patients as well as in certain malignant tumours. The account of alkaline phosphatase isoenzymes in bacteria is far too brief.

The remaining new chapter on the biological significance of isoenzymes is grossly inadequate. There has been a good deal of work in this field particularly in relation to bacteria and plants which ought to have been included in a book described as a comprehensive review of the literature.

As was the case with the first edition, the way this book is arranged calls for a very detailed index. Unfortunately, in practice the very reverse is true and there are a number of significant omissions. For example, there is no reference at all to the discussion of fish lactate dehydrogenase isoenzymes on pages $172-173$. There is also insufficient cross-referencing.

It is doubtful whether there has ever been a perfect book dealing with a scientific topic. Although there is much to criticize, it should be pointed out that this monograph is very lucidly written and contains much relevant information. It can be recommended to readers who are not already engaged in this field. It would not be of much use to the clinician.

A. L. LATNER

\section{Trypanosome and the Fly}

The African Trypanosomiases. Edited

by H. W. Mulligan. Pp. Ixxxviii +950 . (Allen and Unwin: London, March 1971.) £10.00.

THE problems of trypanosomiasis in Africa concern many disciplines. Parasitology, entomology, public health, veterinary science, agriculture and even tourism are all involved. Since the European penetration of Africa in the nineteenth century up to the recent emergence of the new African nations, trypanosomiasis has presented a complex and challenging problem over a wide field of endeavour. In the West, the emphasis has perhaps been chiefly on the human disease, in the East on the infection of animals, though both aspects are important throughout central Africa. The changes in the incidence of the disease have a complex relationship to social and political changes of the period. Proposed methods of control have been many, and sometimes conflicting. With so many disciplines concerned, an overall picture of the situation is opportune.

This book sets out to summarize the varied and voluminous work on trypanosomiasis in Africa from the discovery of the cause of the disease to the present day. A few of the 27 authors of the 43 chapters are still actively engaged on field work in Africa. Many have returned to their native lands. The authors are all English speaking but the work of the other ex-colonial powers is also covered. The names of a few African scientists appear in the references.

For the reader who specializes in none of the many aspects of this subject, the outstanding contribution is Duggan's "Historical Perspective". In 48 pages he outlines the problems of the whole subject, and progress towards their solution. This is the most scholarly contribution; the writing is clear and distinguished. The rest of the book discusses in detail the components of the subject, in four parts. The first two deal with causative organisms and the insect vectors, the third with human trypanosomiasis and the fourth with the trypanosomiases of animals.

This approach has been successful, and has produced a reasonable division of the subject, thereby avoiding overfragmentation into field and laboratory, research and administration or East and West; this could easily have occurred.

Some overlap is inevitable and some differences of opinion-the value of pentamidine prophylaxis, for exampleis acceptable. Agreement on all aspects of this enormous subject is not to be expected.

These chapters detail the laboratory and field work carried out all over
Africa which has resulted in a reasonable degree of control. Much has been done but many questions remain unanswered. It is still hard to foresee the elimination of the trypanosome by the weapons at present available. Will the position deteriorate if the residual insecticides are proscribed ?

The indexing is, perhaps, not wide enough for a reference work of this scope. It is possible to find the work of each author only from the chapter headings. There is no page index of the many references in the bibliography.

I searched for information on the incubation period of the trypanosome in the tsetse fly-a figure basic for a logical approach to control. I found it stated in the chapter on the epidemiology of Gambian sleeping sickness, but could find no reference to the work on which this figure was based in the earlier chapters which cover the basic aspects of the subject, the trypanosome and the fly.

The individual chapters present an admirable summary of the achievements of the period, however, and can stand as a suitable memorial to the authors and their predecessors. The trypanosomiases are not defeated; the information and ideas in this book should provide a base for further attack. M. J. Colbourne

\section{Chromosomes Computed}

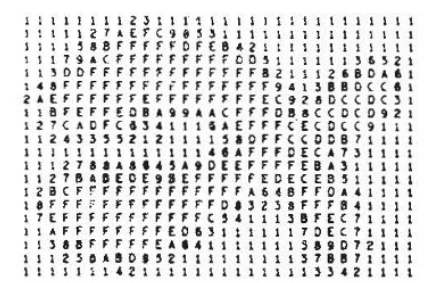

(a)

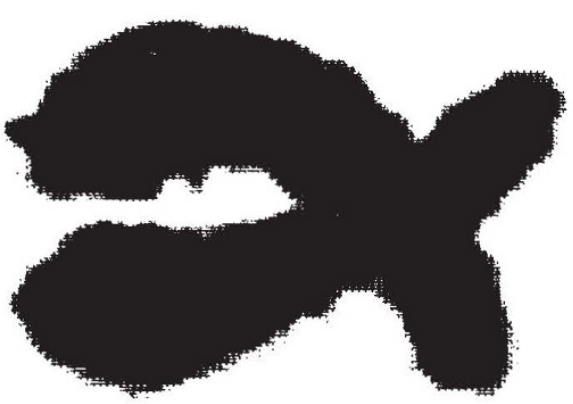

(b)

How to use a computer to recognize a chromosome. Computer methods are being used increasingly to solve pattern recognition problems, both in the physical and biomedical sciences. This illustration, taken from Advances in Computers, Vol. IC (Academic: New York and London, November 1970, £6.75), shows (a) digitization of a photograph of a chromosome provided by a 'FIDAC' flying-spot scanner, and (b) the picture reconstructed. 